\title{
Estudio epidemiológico de histoplasmosis, paracoccidioidomicosis y criptococosis en una zona urbana de Ciudad Guayana, estado Bolivar, Venezuela.
}

\author{
Julman R Cermeño ${ }^{1}$, Ernesto Alayo ${ }^{2}$, Julmery J. Cermeño ${ }^{3}$, Alfredo Calsadilla, \\ Alfredo Rodrígues 5 y Salvador Penna ${ }^{6}$ \\ ${ }^{1}$ Departamento de Parasitología y Microbiología. Escuela de Ciencias de la Salud \\ "Dr. Francisco Battistini Casalta". Universidad de Oriente, Núcleo Bolívar. \\ Ciudad Bolívar. Venezuela. \\ ${ }^{2}$ Hospital "Uyapar". Puerto Ordaz. Venezuela. \\ ${ }^{3}$ Departamento de Medicina Interna. Escuela de Ciencias de la Salud "Dr. Franciseo \\ Battistini Casalta”. Universidad de Oriente, Núcleo Bolívar. Ciudad Bolívar. Venezuela. \\ ${ }^{4}$ Centro Clínico Familia. Puerto Ordaz. Venezuela. \\ ${ }^{5}$ Instituto de Salud Pública del Estado Bolívar. Ciudad Bolívar, Venezuela. \\ ${ }^{6}$ Departamento de Ciencias Fisiológicas. Escuela de Ciencias de la Salud "Dr. Franciseo \\ Battistini Casalta”. Universidad de Oriente, Núcleo Bolívar. Ciudad Bolívar. Venezuela. \\ Palabras clave: histoplasmina; Histoplasma capsulatum; Crytocococcus neoformans; \\ Paracoccidioides; paracoccidioidina.
}

Resumen. Se realizó un estudio prospectivo con el objetivo de determinar la prevalencia de infecciones por Histoplasma capsulatum, Complejo Paracoccidioides spp. y Complejo Cryptoccocus en individuos que viven o trabajan en la Plaza de las Ciencias del Sur, Puerto Ordaz, en el estado Bolívar, Venezuela, donde se refugian aves miǵratorias. Se aplicó una encuesta clínico-epidemiológica y se administraron pruebas intradérmicas de histoplasmina y paracoccidiodina, a todos los sujetos participantes en el estudio. A aquellos individuos sintomáticos respiratorios crónicos, se les realizó una evaluación clínica, radiológica, cultivo micológico y estudio serológico (anticuerpos específicos para Histoplasma capsulatum, Complejo Paracoccidioides spp. y del antígeno capsular del Complejo Cryptococcus spp.). Se aplicaron 632 pruebas intradérmicas a 316 individuos. De estos, sólo 296 (93,7\%) acudieron a la lectura de la pruebas. Los sujetos tenían una media de edad de 40,3 años (rango: 7-76 años). Las manifestaciones clínicas más frecuentes fueron: tos $(\mathrm{n}=49 ; 16,5 \%)$, expectoración $(\mathrm{n}=17 ; 5,7 \%)$ y fiebre $(\mathrm{n}=8 ; 2,7 \%)$. La prueba para la histoplasmina fue positiva 
en el 47,3\% $(n=140)$ y a la paracoccidioidina, en el 32,4\% $(n=96)$. Los factores de riesgos fueron: contacto con palomas $30,4 \%(n=90)$ y visitas a fincas con gallineros 29,1\% $(n=86)$. Se identificaron dos casos de paracoccidioidomicosis pulmonar $(0,7 \%)$ y un caso $(0,3 \%)$ de criptococosis pulmonar. La elevada prevalencia de infección por Histoplasma capsulatum $(47,3 \%)$ y Paracoccidioides spp. $(32,4 \%)$ en ambientes urbanos del estado Bolívar, demuestra la necesidad de promover la búsqueda activa de estos hongos patógenos en quienes tienen síntomas respiratorios crónicos, que no mejoran con terapia antibacteriana y que habitan en zonas con características ambientales y geográficas, propicias para el desarrollo de estos hongos.

\title{
Epidemiological survey of histoplasmosis, paracoccidioidomicosis and cryptococosis in an urban area of Ciudad Guayana, Bolivar state, Venezuela.
}

\section{Invest Clin 2021; 62 (3): 193-207}

Key words: histoplasmin; Histoplasma capsulatum; Crytocococcus neoformans; Paracoccidioides; paracoccidioidine.

\begin{abstract}
The aim of this study was to determine the prevalence of infections by Histoplasma capsulatum, Paracoccidioides spp. and Cryptoccocus complexes. A prospective study was carried out in individuals who live or work in the surroundings of Puerto Ordaz (Plaza de las Ciencias del Sur), where migratory birds refuge in Bolívar state, Venezuela. A clinical-epidemiological survey was applied. Intradermal tests of histoplasmine and paracoccidiodine were administered to all subjects participating in the study. Those individuals with chronic respiratory symptoms underwent a clinical, radiological, mycological culture and serological evaluation (determination of specific antibodies for Histoplasma capsulatum, Paracoccidioides spp. complex and the capsular antigen of the Cryptococcus spp. complex). Six hundred thirty two intradermal tests were applied to 316 individuals. Of these, only 296 (93.7\%) attended the tests evaluation. The subjects had a mean age of 40.3 years-old (range: 7-76 years). The most frequent clinical manifestations were: cough $(\mathrm{n}=49 ; 16.5 \%)$, expectoration $(n=17 ; 5.7 \%)$ and fever $(n=8 ; 2.7 \%)$. Histoplasmine test was positive in $47.3 \%(n=140)$ and paracoccidioidine in $32.4 \%(n=96)$. The risk factors were: contact with pigeons $30.4 \%(n=90)$ and visits to farms with poultry houses $29.1 \%(n=86)$. Two cases of pulmonary paracoccidioidomycosis $(0.7 \%)$ and one case $(0.3 \%)$ of pulmonary cryptococeosis were detected. The high prevalence of Histoplasma capsulatum infection (47.3\%) and Paracoccidioides spp. (32.4\%) in an urban environment of the Bolívar state indicates the need to promote the active search for these pathogenic fungi in people with chronic respiratory symptoms, which do not improve with antibacterial therapy, and live in regions that have environmental and geographical characteristics, leading to such fungal diseases.
\end{abstract}

Recibido: 12-01-2021 Aceptado: 24-07-2021 


\section{INTRODUCCIÓN}

La Histoplasmosis (H) y la Paracoceidioidomicosis (PCM) son las micosis sistémicas, endémicas más frecuentes en Venezuela, causadas por hongos dimórficos, geófilos: Histoplasma capsulatum y especies de Paracoccidioides respectivamente; comunes en América $(1,2)$ y son consideradas un problema importante de salud pública en el estado Bolívar $(3,4)$. La H y la PGM están relacionadas con las condiciones ambientales: humedad, temperatura, pluviometría, características y contenido orgánico del suelo (58), las cuales determinan la distribución de estos hongos en la naturaleza (8).

La mayoría de las personas infectadas con Paracoccidioides spp. desarrollan PCM asintomática o subclínica, que puede progresar hacia una enfermedad con múltiples formas clínicas que dependen de factores en el huésped o ambientales y de la virulencia fúngica (9). La distribución de la paracoccidioidomicosis es heterogénea mostrando alta y baja endemicidad en diferentes áreas y está influenciada por el cambio climático, la migración humana, la expansión de las actividades agrícolas y la construcción de carreteras $(7,10)$.

La criptococosis es una infección causada frecuentemente por un Complejo de especies: Cryptococcus neoformans y C. gattii, el cual ocasiona enfermedad en humanos y animales $(11,12)$. Son levaduras encapsuladas que se reproducen por gemación y que pueden ocasionar enfermedad tanto en personas inmunocompetentes como inmunocomprometidas. Frecuentemente estas levaduras afectan al Sistema Nervioso Central $(13,14)$.

En Venezuela, la criptococosis es la cuarta micosis profunda sistémica diagnosticada (2) y, frecuentemente, causa meningitis sobre todo en pacientes con infección por el Virus de Inmunodeficiencia Humana (2). La prevalencia de infecciones fúngicas sistémicas en individuos con infección por el virus de inmunodeficiencia humana en el estado Bolívar es del 35,7\%, siendo la candidiasis oro-faríngea, histoplasmosis, paracoccidioidomicosis y criptococosis las más prevalentes (3).

La infección por H. capsulatum y por el Complejo Cryptococcus spp. es típicamente adquirida por la inhalación de propágulos o conidios del hongo, después de la manipulación de suelo contaminado con excrementos de aves $(8,13,15-17)$. La manifestación clínica de la enfermedad varía desde una infección asintomática o una enfermedad leve, similar a la gripe, a una forma diseminada que puede afectar cualquier tejido $(6,17)$. La mayoría de las personas infectadas por Histoplasma capsulatum no presentan síntomas o cursan con enfermedad leve que no es reconocida como histoplasmosis. De hecho, el 95-99\% de las infecciones primarias no se reconocen o se detectan en huéspedes inmunológicamente indemnes en áreas endémicas (6).

La llegada de las aves migratorias, especialmente a la Plaza de las Ciencias del Sur, en Puerto Ordaz, Estado Bolívar, se considera un importante movimiento migratorio de golondrinas en el planeta. Cada año, llegan aves procedentes del sur del continente americano, casi 500 mil individuos de la especie Progne tapera fusca (Golondrina de río) y 3 mil de Tyrannus savana (Atrapamoseas tijereta) que, escapando del frío austral, encuentran refugio en las frondosas ceibas de la Plaza de las Ciencias del Sur, ubicada en la calle Caicara del centro de Puerto Ordaz. Estas aves eliminan gran cantidad de excrementos en la Plaza y sus alrededores $(18,19)$, lo que podría constituir un riesgo para la salud, debido a que éstas pueden ser consideradas reservorios potenciales de agentes patógenos virales, bacterianos, parasitarios y fúngicos y posibles transmisores de los mismos (20-25).

La PCM comparte algunas características epidemiológicas y clínicas semejantes a la $\mathrm{H}$ y criptococosis en una misma región $(3,4)$. En los hospitales, tanto de Puerto Ordaz como de Ciudad Bolívar (centro de refe-

Vol. 62(3): 193 - 207, 2021 
rencia del estado), se ha incrementado la casuística de micosis sistémicas en los últimos 10 años; sin embargoo, los casos no han sido publicados. En tal sentido, se decidió realizar la presente investigación, motivados por la solicitud realizada por médicos especialistas y líderes de la comunidad, quienes refirieron un incremento de casos de histoplasmosis y paracoccidioidomicosis en sujetos procedentes del centro de Puerto Ordaz. El estudio tiene la finalidad de determinar la prevalencia de la infección por Histoplasma capsulatum, del Complejo Paracoccidioides spp y Cryptococcus en individuos que viven en las urbanizaciones de los alrededores de la Plaza de las Ciencias del Sur, donde se refugian estas aves migratorias en el estado Bolívar.

\section{MATERIAL Y MÉTODOS}

Se realizó un estudio clínico-epidemiológico de tipo descriptivo, transversal y prospectivo.

\section{Área de estudio}

Se incluyeron a aquellos individuos que viven o trabajan en el centro de Puerto Ordaz, las urbanizaciones ubicadas alrededor de la Plaza de las Ciencias del Sur $\left(8^{\circ} 18^{\prime} 59.0\right.$ ”N 62 42'55.0”W), municipio Caroní, estado Bolívar, Venezuela; aproximadamente $5 \mathrm{Km}$ a la redonda: Urbanización Alta Vista Sur, La Querencia, Chilemex, Villa Alianza, La Corniza, Los Saltos y Arivana.

\section{Inspección visual}

Con la ayuda de un especialista en Ornitología y Biología, se realizó una visita al área con el fin de evidenciar restos de excretas de aves, la presencia de las aves migratorias y las especies involucradas. También se consideraron factores ambientales asociados que interfieren en el proceso salud enfermedad. Para ello, se realizaron desplazamientos en todo el sector en un diámetro de $5 \mathrm{Km}$ a la redonda.

\section{Muestra}

La muestra estuvo constituida por 316 individuos pertenecientes a la comunidad que viven o que trabajan alrededor de la Plaza de las Ciencias del Sur, en Puerto Ordaz, estado Bolívar, quienes dieron, voluntariamente, su consentimiento informado para participar en el estudio. Se respetaron los principios éticos para la investigación médica en seres humanos, siguiendo los lineamientos de la Declaración de Helsinki (Actualizada en 2008). El estudio fue realizado entre los meses de agosto del año 2014 a febrero 2015.

\section{Recolección de datos}

Una vez obtenido el consentimiento informado se procedió a realizar una encuesta para la recolección de datos clínicosepidemiológícos y demográficos (sexo, edad, procedencia, ocupación), antecedentes de importancia (enfermedades cardiovasculares, diabetes, asma bronquial, bronquitis, alergias), hábitos psicobiológicos (hábitos tabáquicos, alcohólicos, uso de drogas de abuso) manifestaciones clínicas, tiempo en la zona, así como también factores de riesgoo (contacto con murciélagos, perros, gatos, palomas, pollos, gallinas, aves migratorias $\mathrm{u}$ otras, visita a fincas con aves, visitas a cuevas, realizar labores de agricultura), entre otros que interfieren en el proceso saludenfermedad. A los individuos sintomáticos respiratorios crónicos (con sintomatología mayor a tres meses de evolución) se les realizó la evaluación clínica, radiografía de tórax postero-anterior y lateral, estudio micológico de la secreción respiratoria, tinción para micobacterias, demostración de anticuerpos específicos para Histoplasma capsulatum, Complejo Paracoccidioides spp. y antígeno capsular del Complejo Cryptococcus spp. en suero. Toda la información referida fue recogida en una ficha elaborada para tal fin.

El estudio radiológico se efectuó en una clínica privada de Puerto Ordaz. 


\section{Pruebas intradérmicas}

Se realizaron dos pruebas intradérmicas: histoplasmina y paracoccidiodina para la investigación de las infecciones causadas por Histoplasma capsulatum y especies de Paracoccidioides, respectivamente en todos los sujetos que participaron en el estudio.

Para la realización de las pruebas intradérmicas se emplearon los antígenos elaborados por el Instituto de Biomedicina, de la Universidad Central de Venezuela. La histoplasmina, un antígeno metabólico obtenido de la fase filamentosa del Histoplasma capsulatum var capsulatum, se presenta en una dilución 1:1000 y la paracoccidiodina, un polisacárido obtenido de la fase levaduriforme de 8-9 aislados del Complejo Paracoccidioides spp., en una dilución 1:20, siguiendo las recomendaciones de Fava-Netto y Raphael (26).

En los individuos sintomáticos respiratorios las pruebas intradérmicas fueron realizadas inmediatamente después de la toma de muestra de sangre venosa para la determinación de anticuerpos específicos contra el Complejo Paracoccidioides spp, Histoplasma capsulatum y antígeno capsular de Cryptococcus spp.

Para la realización de las pruebas intradérmicas, a cada sujeto participante del estudio se le inocularon $0,1 \mathrm{~mL}$ de histoplasmina y paracoccidioidina en la cara anterior del tercio proximal del antebrazo izquierdo y derecho, respectivamente. La zona de inoculación fue identificada con una marea. Las lecturas de las pruebas intradérmicas fueron realizadas a las 24 y 48 horas después de aplicación de la prueba. La prueba intradérmica se consideró positiva cuando se observó un área de induración con un diámetro de $5 \mathrm{~mm}$ o más en el lugar de inoculación (27-30).

\section{Muestra de esputo}

Se recolectaron muestras de esputo solo en aquellos individuos que presentaron tos productiva crónica al momento del estudio. Estas fueron recogidas en envases estériles y transportados en cavas con hielo hasta el Departamento de Parasitología de la Escuela de Ciencias de la Salud "Dr. Franciseo Battistini Casalta" de la Universidad de Oriente, Núcleo Bolívar y conservadas a $4^{\circ} \mathrm{C}$ hasta su procesamiento. Las muestras de esputo fueron concentradas mediante centrifugación a $2.500 \mathrm{x} g$ durante 20 minutos y el sedimento fue re suspendido con $2 \mathrm{~mL}$ de tampón fosfato. A cada una de las muestras de esputo se les realizó un examen directo con $\mathrm{KOH}$ al $20 \%$ y con tinta china. Se realizaron tres frotis con el sedimento para llevar a cabo las coloraciones de Ziehl-Neelsen (Z-N), tinción con Giemsa y tinción con metenamina argéntica. Además, parte del sedimento $(0,5$ $\mathrm{mL})$ fue sembrado por duplicado en medios de Agar glucosado de Sabouraud (Oxoid ${ }^{\circledR}$ ) más cloranfenicol, medio agar de semillas de alpiste (Guizotia abyssinica), medio agar de semillas de girasol (Helianhus annus) y agar cerebro corazón $\left(\right.$ Oxoid $\left.^{\circledR}\right)$. Los cultivos fueron realizados por duplicado e incubados a $25^{\circ} \mathrm{C}$ y a $35^{\circ} \mathrm{C}$. Estos fueron observados hasta por 8 semanas.

La identificación de los hongos fue realizada por las técnicas estándares de laboratorio, aplicando los siguientes criterios para todos los agentes: las estructuras observadas en el examen directo, características macroscópicas y microscópicas de la colonia. Además, se aplicaron otros criterios, los indicados por Kwong-Chung y Bennett (31) y Hoog y col. (32).

Las colonias levaduriformes desarrolladas en los medios selectivos fueron sometidas a la identificación de la cápsula, prueba de la ureasa, test de resistencia a la actidiona y se empleó el sistema API 20C y API ID 32C (Biomerieux $^{\circledR}$, Francia). La interpretación de las pruebas bioquímicas se realizó mediante el software Apiweb de Biomerieux ${ }^{\circledR}$. Como cepa control se incluyeron: ATCC 22019 (Candida parapsilosis), ATCC 6258 (Candida krusei), ATCG 90028 (Candida albicans). Los aislamientos identificados como Cryptococcus spp. fueron sembrados en medio con L-canavanina glicina azul de bromotimol 
(CGB) (Sigma ${ }^{\circledR}$ ) para la diferenciación entre C. neoformans y C. gattii (33-35).

Los cultivos fueron manejados dentro de una campana de flujo laminar, con un nivel de bioseguridad estricto, manipulando los cultivos con precaución, trabajando con todas las medidas adecuadas de bioseguridad.

\section{Serología de hongos}

La serología para hongos, determinación de anticuerpos específicos para Histoplasma capsulatum, Complejo Paracoccidioides spp. y del antígeno capsular del Complejo Cryptococcus spp., fueron realizadas solo a aquellos sujetos con síntomas respiratorios crónicos en el momento del estudio. Para ello, se les tomó muestra de sanǵre venosa de la vena cubital en tubos estériles al vacío (Vacutainer ${ }^{\circledR}$ ). Media hora después de su obtención, las muestras fueron centrifugadas a $3.500 \mathrm{~g} \mathrm{x} 10$ minutos, obtenido el suero, fraccionado en alícuotas de $1 \mathrm{~mL}$ y conservados a menos $20^{\circ} \mathrm{C}$ hasta su procesamiento en el Departamento de Parasitología de la Escuela de Ciencias de la Salud "Dr. Francisco Battistini Casalta" de la Universidad de Oriente, Núcleo Bolívar.

Para la demostración de anticuerpos específicos contra el Complejo Paracoccidioides spp. e Histoplasma capsulatum, se empleó la técnica de doble difusión en gel de agarosa (36).

Los antígenos fúngíicos del Complejo Paracoccidioides spp. fueron obtenidos a partir del sobrenadante del cultivo del hongo en su fase micelial y fabricados por el Instituto de Biomedicina, UCV, Caracas (Lote 33922210). El antígeno de Histoplasma capsulatum (Lote 112AH5) y el suero control de Histoplasma (Lote H60110), procedían de Immy Immunodiagnostics, Inc., USA ${ }^{\circledR}$.

Se emplearon sueros específicos (controles) obtenidos de sujetos con diagnóstico de paracoccidioidomicosis, confirmado mediante biopsia. Se consideraron positivos aquellos sueros que mostraron bandas o líneas de identidad específica entre el pozo de la muestra del suero y el pozo del antígeno marcador para cada una de las micosis investigadas. La detección de Banda $\mathrm{H}$ y/o Banda $\mathrm{M}$ fue considerada como prueba positiva para $H$. capsulatum. La presencia de banda o líneas control sin la observación de bandas o líneas entre el pozo de la muestra del suero y el pozo del antígeno fue interpretada como un resultado negativo (37).

La determinación del antígeno capsular de Cryptococcus spp. fue realizada mediante el ensayo inmunoenzimático $\left(\right.$ Premier $^{\circledR}$ ) de Meridian Bioscience, Inc. interpretando los resultados según los criterios descritos por el fabricante.

\section{Análisis estadístico}

Se emplearon estadígrafos descriptivos. Las variables cualitativas se expresaron indicando las frecuencias absolutas y la proporción de cada una de las eategorías; las variables cuantitativas se expresaron como medias y desviaciones estándar. Se empleó la prueba de $\mathrm{Ji}^{2}$ y la prueba Exacta de Fisher para comparar las variables cualitativas, considerando un nivel de significancia del 95\%. Se utilizó el paquete estadístico SPSS versión 21.0 para Ordenador IBM.

\section{RESULTADOS}

Se aplicaron 632 pruebas intradérmicas a 316 individuos quienes aceptaron voluntariamente participar en el estudio. De éstos, solo $296(93,7 \%)$ acudieron a la lectura de la prueba a las 24 y 48 horas, respectivamente. Veinte individuos no acudieron a las lecturas de las pruebas cutáneas $(6,3 \%)$.

La encuesta demostró que la mayoría de los sujetos vivían en Puerto Ordaz $(n=256$; $86,5 \%)$ y procedían predominantemente de la Parroquia Cachamay $(n=124 ; 41,8 \%)$, Unare $(n=68 ; 22,9 \%)$ y Universidad $(n=64$; 21,6\%) (Ver Tabla I). La mayoría era del sexo femenino $(n=177 ; 59,8 \%)$. Los sujetos estudiados eran docentes $(n=163 ; 55,1 \%)$, administrativos $(n=28 ; 9,4 \%)$, comerciantes $(n=$ $43 ; 14,5 \%)$, obreros $(n=35 ; 11,8 \%)$, estudiantes $(n=25 ; 8,4 \%)$ y chofer $(n=2 ; 0,6 \%)$. 
TABLA I

DISTRIBUCIÓN DE FREGUENCIAS SEGÚN SEXO, EDAD Y PROCEDENCIA DE LOS INDIVIDUOS EVALUADOS. CIUDAD GUAYANA. MUNICIPIO CARONÍ. ESTADO BOLÍVAR. VENEZUELA.

\begin{tabular}{lcc}
\hline Variables & $\mathrm{n}$ & $\%$ \\
\hline Sexo & & \\
$\quad$ Femenino & 177 & 59,8 \\
$\quad$ Masculino & 119 & 40,2 \\
Edad (años): : $\bar{x}=40,3+11,7$ & & \\
1 -10 & 15 & 5,0 \\
$11-20$ & 40 & 13,5 \\
$21-30$ & 68 & 22,9 \\
$31-40$ & 34 & 11,4 \\
$41-50$ & 71 & 23,9 \\
$51-60$ & 23 & 7,7 \\
$61-70$ & 35 & 11,8 \\
$71-80$ & 10 & 3,3 \\
Procedencia (Parroquias) & & \\
Cachamay & 124 & 41,8 \\
Unare & 68 & 22,9 \\
Universidad & 64 & 21,6 \\
Simón Bolívar & 11 & 3,7 \\
Dalla Costa & 10 & 3,4 \\
Chirica & 8 & 2,7 \\
11 de Abril & 2 & 0,7 \\
Vista al Sol & 8 & 2,7 \\
Yocoima & 1 & 0,3 \\
\hline
\end{tabular}

Las personas evaluadas tenían una media de edad de 40,3 años, (rango: 7-76 años). El grupo de edad más frecuente fue de 41 a 50 años $(\mathrm{n}=71 ; 23,9 \%)$.

Dentro de los antecedentes patológicos de importancia destacan: hipertensión arterial $(\mathrm{n}=56 ; 18,9 \%)$, diabetes mellitus $(n=36 ; 12,2 \%)$, asma bronquial $(n=35$; $11,8 \%)$, bronquitis recurrentes $(n=23$; $7,8 \%)$ y alergias $(n=13 ; 4,4 \%)$. Dentro de los hábitos psicobiológicos, se evidenció que 86 sujetos tenían hábitos alcohólicos ocasiona- les $(29,2 \%), 39$ eran fumadores (13,2\%) y 11 realizaban labores de aǵricultura ocasional $(3,7 \%)$. El resto de los individuos no refirieron antecedentes patológicos ni hábitos psicobiológicos de importancia médica.

Las manifestaciones clínicas señaladas fueron: tos $(\mathrm{n}=49 ; 16,5 \%)$, expectoración $(\mathrm{n}=17 ; 5,7 \%)$, fiebre $(\mathrm{n}=8 ; 2,7 \%)$, malestar en la garganta $(n=5 ; 1,7 \%)$, dolor torácico $(n=3 ; 1,0 \%)$, disnea $(n=1 ; 0,3 \%)$ y cefalea $(\mathrm{n}=1 ; 0,3 \%)$.

La histoplasmina fue positiva en el $47,3 \%(n=140)$ y la paracoccidioidina en el $32,4 \%(\mathrm{n}=96)$ de los sujetos evaluados (Tabla II). Solo 77 sujetos mostraron, simultáneamente, ambas pruebas positivas $(26,0 \%)$. La infección para el Complejo Paracoccidioides spp. fue mayor en el sexo masculino $(p=0,0001)$, sin relación con la edad; sin embargoo, la infección por Histoplasma capsulatum fue superior en el sexo femenino $(\mathrm{p}=0,011)$, y se observó más frecuentemente en mayores de 35 años $(p=0,001)$.

La frecuencia de infección por Complejo Paracoccidioides spp. y/o Histoplasma capsulatum resultó independiente del tiempo de permanencia en Puerto Ordaz y del tiempo que llevaban viviendo en las diferentes parroquias $(p>0,05)$.

El mayor porcentaje de intradermorreacción positiva a histoplasmina se observó en los grupos de edades comprendidos entre 41 a 50 años $(\mathrm{p}=0,0001)$ y con la prueba de paracoccidioidina, en el grupo de 31 a 40 años $(p>0,05)$.

Los factores de riesgos se muestran en la Tabla III, donde se observa que los mismos no están asociados a la infección por hongos $(\mathrm{p}>0,05)$, solo la visita a campos con gallineros se asoció, con baja significancia, a la infección por Paracoccidioides spp. $(\mathrm{p}=0,05)$. Ningún individuo mencionó el contacto con aves migratorias, ni con sus excretas en la Plaza de la Plaza de las Ciencias del Sur.

Con relación a las manifestaciones clínicas, solo la tos crónica se asoció con infecciones por Paracoccidioides spp. $(\mathrm{p}=0,043)$. 
TABLA II

PRUEBAS INTRADÉRMICAS SEGÚN SEXO Y EDAD DE LOS INDIVIDUOS EVALUADOS. GIUDAD GUAYANA. MUNICIPIO CARONÍ. ESTADO BOLÍVAR.VENEZUELA.

\begin{tabular}{|c|c|c|c|c|c|c|}
\hline \multirow[t]{2}{*}{ Variables } & \multicolumn{2}{|c|}{ Histoplasmina } & \multirow[t]{2}{*}{$\mathrm{p}^{*}$} & \multicolumn{2}{|c|}{ Paracoccidioidina } & \multirow[t]{2}{*}{$\mathrm{p}$} \\
\hline & Positiva (\%) & Negativa (\%) & & Positiva (\%) & Negativa (\%) & \\
\hline \multicolumn{7}{|l|}{ Sexo } \\
\hline Femenino & $75(25,3)$ & $102(34,4)$ & 0,011 & $45(15,2)$ & $132(44,5)$ & \\
\hline Masculino & $65(21,9)$ & $54(18,2)$ & & $51(17,2)$ & $68(22,9)$ & 0,0001 \\
\hline Total & $140(47,3)$ & $156(52,7)$ & & $96(32,4)$ & $200(67,6)$ & \\
\hline \multicolumn{7}{|l|}{ Edad (años) } \\
\hline $1-10$ & $1(0,3)$ & $14(4,7)$ & & $1(0,3)$ & $14(4,7)$ & \\
\hline $11-20$ & $9(3,0)$ & $31(10,4)$ & & $5(1,6)$ & $35(11,8)$ & \\
\hline $21-30$ & $20(6,7)$ & $48(16,2)$ & & $15(5,0)$ & $53(17,9)$ & \\
\hline $31-40$ & $33(11,1)$ & $1(0,3)$ & & $17(5,6)$ & $17(5,4)$ & \\
\hline $41-50$ & $37(12,5)$ & $34(11,4)$ & 0,0001 & $16(5,4)$ & $55(18,5)$ & \\
\hline $51-60$ & $17(5,7)$ & $6(2,0)$ & & $14(4,7)$ & $9(3,0)$ & \\
\hline $61-70$ & $20(6,7)$ & $15(5,0)$ & & $10(3,3)$ & $25(8,4)$ & \\
\hline $71-80$ & $6(2,0)$ & $4(1,3)$ & & $6(2,0)$ & $4(1,3)$ & \\
\hline Total & $140(47,3)$ & $156(52,7)$ & & $96(32,4)$ & $200(67,6)$ & \\
\hline
\end{tabular}

* Se refiere a la prueba de Ji al cuadrado (un valor de $\mathrm{p}<0,05$ fue considerado significativo).

TABLA III

PRUEBAS INTRADÉRMICAS SEGÚN FACTORES DE RIESGO DE LOS INDIVIIDUOS EVALUADOS. GIUDAD GUAYANA. MUNICIPIO CARONÍ. ESTADO BOLÍVAR.VENEZUELA.

\begin{tabular}{|c|c|c|c|c|c|c|c|}
\hline \multirow{2}{*}{$\begin{array}{c}\text { Variables } \\
\text { Factores de riesgo* }\end{array}$} & \multirow[b]{2}{*}{$\begin{array}{c}\mathrm{n} \\
(\%)\end{array}$} & \multicolumn{2}{|c|}{ Histoplasmina } & \multirow[t]{2}{*}{ Valor $\mathrm{p}$} & \multicolumn{2}{|c|}{ Paracoccidioidina } & \multirow[t]{2}{*}{ Valor $\mathrm{p}^{* *}$} \\
\hline & & $\begin{array}{c}\text { Positiva } \\
\text { (\%) }\end{array}$ & $\begin{array}{c}\text { Negativa } \\
(\%)\end{array}$ & & $\begin{array}{l}\text { Positiva } \\
\text { (\%) }\end{array}$ & $\begin{array}{l}\text { Negativa } \\
\text { (\%) }\end{array}$ & \\
\hline Contacto con palomas & $90(30,4)$ & $38(12,8)$ & $52(17,5)$ & & $28(9,4)$ & $62(20,9)$ & \\
\hline $\begin{array}{l}\text { Visita a fincas } \\
\text { con gallineros }\end{array}$ & $86(29,1)$ & $43(14,5)$ & $43(14,5)$ & & $59(19,9)$ & $27(9,1)$ & 0,05 \\
\hline Contacto con gallinas & $43(14,5)$ & $20(6,7)$ & $23(7,7)$ & & $17(5,7)$ & $26(8,7)$ & \\
\hline Visitas a Cuevas & $35(11,8)$ & $15(5,1)$ & $20(6,7)$ & & $12(4,1)$ & $23(7,7)$ & \\
\hline Contacto con perros & $31(10,5)$ & $17(5,7)$ & $14(4,7)$ & & $8(2,7)$ & $23(7,7)$ & \\
\hline Contacto con murciélagoos & $25(8,4)$ & $14(4,7)$ & $11(3,7)$ & & $12(4,1)$ & $13(4,3)$ & \\
\hline
\end{tabular}

*Los individuos tenían más de un factor de riesgó. * Se refiere a la prueba de Ji al cuadrado (un valor de $\mathrm{p}<0,05$ fue considerado signnificativo). 
Se evaluaron clínicamente 11 individuos sintomáticos respiratorios crónicos, todos de género masculino $(3,7 \%$ de la población total) y ninguno de ellos tenía antecedentes psicobiológicos de importancia médica. El examen físico fue normal y solo dos de ellos, masculinos, tenían escasos estertores y roncus en ambas bases pulmonares. La radiografía de tórax mostró alteraciones en 9 individuos: reforzamiento hiliar derecho $(n=2)$, reforzamiento hiliar bilateral $(\mathrm{n}=1)$, aumento de radiotransparencia ambos pulmones $(n=1)$, aumento de la trama bronquial $(n=3)$, trama bronquial reforzada, paredes bronquiales engrosadas, infiltrado peribronquítico e intersticial difuso $(n=2)$, el resto fue normal.

$\mathrm{El}$ examen directo de esputo con $\mathrm{KOH}$ al 20\%, la tinción con Giemsa y la coloración de Grocott demostraron la presencia de levaduras multigemantes del Complejo Paracoccidioides spp en 2 casos $(0,7 \%)$ y el examen directo con la tinta china evidenció levaduras encapsuladas del Complejo Cryptococcus sp. en 1 solo caso (0,3\%). Estos sujetos mostraron alteraciones radiológicas: trama bronquial reforzada, paredes bronquiales engrosadas, infiltrado peribronquítico, e intersticial difuso y aumento de radiotransparencia de ambos pulmones. Además presentaron las siguientes manifestaciones clínicas: tos, expectoración, disnea, así como dolor torácico y fiebre compatibles de una PCM pulmonar crónica y criptococosis pulmonar. En los cultivos micológicos de esputo fueron aislados los hongos Paracoccidioides spp. y C. neoformans.

En ningún caso se evidenció serología positiva para Histoplasma capsulatum, Complejo Paracoccidioides spp. ni para el Complejo Cryptococcus spp. En las coloraciones de Z-N no fueron observadas micobacterias.

La inspección de la Plaza de las Ciencias del Sur mostró que la limpieza es deficiente, así como sus alrededores. Se evidenció excremento de aves migratorias en todo el piso creando una capa de $2-3 \mathrm{~cm}$ de espesor, excretas en las hojas de arbustos, en el tallo y hojas de los árboles; de igual modo, en la calle sobre el asfalto, a un metro del borde externo de la plaza. Se observó la presencia de moscas (Musca domestica) y un olor fuerte a ácido que irritaba las fosas nasales. En las urbanizaciones ubicadas a los $5 \mathrm{Km}$ alrededor de la plaza se observaron frecuentemente palomas cercanas a los sitios donde quedaban restos de alimentos, en los techos de los edificios, cercanos a los aires acondicionados, en los techos de los puestos de ventas de revistas y algunos lugares de venta de alimentos. En toda el área se observaron abundantes desperdicios de alimentos y basura orgánica e inorgánica.

Los responsables de la investigación consignaron informe a los coordinadores de los consejos comunales y a la Unidad Territorial del Ministerio para Ciencia, Tecnología e Innovación de Ciudad Guayana, dando las recomendaciones pertinentes.

\section{DISCUSIÓN}

Este estudio demuestra una elevada prevalencia de infección por Histoplasma capsulatum $(47,3 \%)$ y por el Complejo $P a$ racoccidioides spp., $(32,4 \%)$ en los sujetos evaluados, similar al estudio realizado en la sede de la Casa de las Doce Ventanas de la UNEG en Ciudad Bolívar (27), aunque en esta última sede la prevalencia fue superior para ambas infecciones $(61,5 \%$ y $46,2 \%$, respectivamente). Este es el primer estudio de esta índole realizado en un área urbana del municipio Caroní del estado Bolívar.

Estudios previos, realizados en comunidades suburbanas del estado Bolívar, señalan una prevalencia inferior para ambas infecciones, en comparación con la encontrada en esta investigación. En San Félix, la prevalencia encontrada fue del 7,6\% para $H$. capsulatum y 10,2\% para el Complejo Paracoccidioides spp. (38); en Monte Ralo, área rural y agrícola del estado, la seroprevalencia fue de $8,5 \%$ y $11,3 \%$ para cada una de las infecciones (39) y en Upata fue de $25,7 \%$ y $19,6 \%$, respectivamente (40). 
Estos resultados demuestran un mejor conocimiento epidemiológico de las infecciones causadas por el Complejo Paracoccidioides spp. e H. capsulatum, hongos patógenos endémicos en el estado Bolívar; observándose una mayor prevalencia de las infecciones en áreas urbanas que en áreas rurales o suburbanas, a pesar de que 11 individuos desempeñaban labores agrícolas en forma ocasional, las cuales se conocen que juegan una papel importante en la adquisición de la infección ocasionada por el Complejo Paracoccidioides spp.

En Ciudad Bolívar, municipio Angostura del Orinoco, sólo existen dos estudios previos de prevalencia de la infección causada por estos hongos patógenos en áreas urbanas uno realizado en la Casa de Las Doce Ventanas de La Universidad Nacional Experimental de Guayana (UNEG) (27) y otro realizado en la Sede 5 de julio de la misma institución (4). Esto demuestra una variabilidad en la frecuencia de la infección por estos patógenos primarios en los individuos que viven en diferentes áreas geográficas del estado Bolívar. Quizás esa elevada prevalencia en el área urbana obedece a las diferentes movilizaciones de tierra por construcciones de nuevas edificaciones y viviendas, a invasiones de terrenos con construcciones improvisadas que se observan en diferentes zonas del municipio Caroní, así como también a la presencia de aves migratorias y/o de palomas que frecuentan casas, edificios, plazas, avenidas, calles, mercados, hospitales, entre otros, donde quedan restos de alimentos (venta ambulantes de maíz y comida rápida) y/o basura que se observan en toda la ciudad, sin ninguna preocupación por parte de las autoridades competentes.

Los hallazgos obtenidos en esta investigación sugieren que en los individuos procedentes del área geográfica estudiada y que hacen vida en Puerto Ordaz presentan, simultáneamente, infecciones del Complejo Paracoccidioides spp., Histoplasma capsulatum y Cryptococcus spp.
Hubo coexistencia de la infección por ambos hongos en el 26,0\% $(n=77)$, similar a lo demostrado en Brasil por Silva-Vergara y Martínez (41) quienes efectuaron un análisis de asociación similar entre las intradermorreacciones positivas a la paracoccidioidina y histoplasmina en cultivadores de café. Estos hallazgoos podrían explicarse debido a la coexistencia de ambos aǵentes micóticos en la región objeto de la investigación, tal como ocurrió en el presente estudio.

En otras regiones de Venezuela, donde la histoplasmosis y la paracoccidioidomicosis son endémicas, se han señalado prevalencias de infección variables, como en la Sierra de Perijá (estado Zulia), Paracotos (estado Miranda), Isla de la Culebra (estado Carabobo) y Sierra de San Luis (estado Falcón), donde la positividad se estima alrededor del $60 \%(42,43)$.

En Latinoamérica, la prevalencia de infecciones por $H$. capsulatum y el Complejo Paracoccidioides spp. es variable $(1,28-$ 30,41,44). En regiones de Argentina se han descrito áreas altamente prevalentes de histoplasmosis ( $>30 \%$ de los individuos positivos a histoplasmina) en Vipos, La Toma y Coromoto (Trancas-Tucumán) y 10,2\% para el Complejo Paracoccidioides spp. (45). En un área rural de Minas Gerais en Brasil se describe un $45,8 \%$ de infección para Paracoccidioides spp (30) y 17,5\% para Histoplasma (46). Sin embargo, en este estudio sólo $11,8 \%$ de los individuos evaluados $(n=35)$ tenían el antecedente de haber visitado cuevas, lo que implica una exposición mayor al hongo en ambientes abiertos y que no sólo el antecedente de visitas a cuevas constituye un factor de riesgo importante en la adquisición de esta infección.

El mayor porcentaje de positividad para la paracoccidioidina e histoplasmina se observó en el grupo de los mayores de 35 años, similar a lo descrito en otras investigaciones en la región $(4,38)$.

Las infecciones por Histoplasma capsulatum, Complejo Paracoccidioides spp. y 
Cryptococcus spp., son endémicas en el estado Bolívar $(3,4)$ probablemente sea debido al bioclima tropical húmedo del estado, a las condiciones de humedad, vegetación, temperatura y características del suelo $\left(27^{\circ} \mathrm{C}\right.$ $31^{\circ} \mathrm{C}$, pluviosidad entre $500-2500 \mathrm{~mm} /$ año, humedad entre $67-87 \%$ y suelos con alto contenido orgánico) que determinan la distribución de estos hongos en la naturaleza $(5-8,15,16,30)$ y, posiblemente, a la presencia de aves (golondrinas y palomas) $\mathrm{u}$ otros reservorios en la ciudad (15). Ello implica, que todo personal de salud debe estar alerta ante la presencia de síntomas respiratorios crónicos en pacientes que no responden a los antimicrobianos y que procedan de esta región geográfica.

Hubo dos casos de paracoceidioidomicosis pulmonar y un caso de criptococosis pulmonar. Es importante señalar que cuando se hacen estudios epidemiológicos en busca activa de infecciones por hongos sistémicos, es frecuente la detección de personas enfermas y en especial de esta región $(4,27)$.

En los alrededores de la Plaza de las Ciencias habitan palomas, cuyo papel como portadoras de hongos patógenos fue establecido por Emmons en 1955, quien aisló C. neoformans de las excreciones en zonas urbanas, siendo el primero en establecer la relación existente entre el microorganismo $\mathrm{y}$ las heces de estas aves (34). Actualmente, numerosos estudios de casi todo el mundo han demostrado que las deposiciones de palomas son un importante reservorio del Complejo Cryptococcus spp. (47-51) y suele aislarse el hongo en las deyecciones acumuladas de estas aves tanto frescas como secas en palomares, aleros de edificios, áticos o balcones de casas abandonadas, donde suelen pernoctar (50). Histoplasma capsulatum también ha aislado de los excrementos de estas aves (50-52).

Parece que la alta concentración de creatinina en el estiércol de paloma favorece el crecimiento de los criptococos; pero además, las heces de pichón, brindan otras características como un ambiente alcalino, hiperosmolar y rico en muchos compuestos nitrogenados, además de la creatinina (34).

La permanencia de la levadura en deyecciones de palomas a la sombra, húmedas o desecadas, puede ser de hasta más de dos años y parece que la capacidad de las especies patógenas de Cryptococcus para producir pigmentos melanoides no sólo les permite sobrevivir a la radiación solar, sino que pueden llegar a utilizar las radiaciones como energía metabólica. Posiblemente, este hecho les permite sobrevivir hasta que los excrementos se convierten en polvo. El polvo vehicula levaduras de solo 1-2 micras de diámetro, pudiendo alcanzar fácilmente el espacio alveolar al ser inhaladas (34).

Se recomendó a personas de la zona y con enfermedades inmunosupresoras, no frecuentar los alrededores de esta plaza, evitar el paseo de mascotas (perros, gatos) y la vigilancia de los niños para evitar su contacto con excretas de aves. Además, se señaló que es necesario evitar la acumulación de las excretas de aves migratorias en la Plaza. Para ello, es necesario realizar limpieza exhaustiva y diaria del lugar. Las personas que limpian la plaza deberían conocer los riesgos a los que podrían exponerse si no usan las medidas de prevención al manipular los excrementos de las aves.

Evitar que la limpieza de la plaza sea realizada por ancianos, ya que su sistema inmunológico está debilitado. Educar a la población sobre la necesidad de no proporcionarle alimentos a las palomas en los parques, jardines ni plazas.

No debe emplearse el excremento de las aves migratorias como abono sin un tratamiento previo que garantice la seguridad de los consumidores.

Las autoridades competentes deben realizar un control exhaustivo y vigilancia de la venta de comida ambulante (ubicación, hiǵiene y seguridad de alimentos) así como la venta de maíz tierno o masa de maíz en sitios improvisados: aceras, calles, avenidas, redomas, entre otros ya que los restos de alimentos, desperdicios y granos son dejados 
en el lugar de la venta y constituyen el alimento principal de las palomas en medios urbanos. Asimismo, los restos de alimentos suministrados a los perros en las casas o edificios deben recogerse y eliminarse después de la alimentación del mismo, para que no constituya el alimento principal de las aves.

Fomentar la investigación de búsqueda de virus, bacterias, parásitos y hongos, de interés en salud pública, en las aves migratorias. Establecer vigilancia sanitaria de las aves migratorias, para ello deben capturarse algunos ejemplares y someterlos a una analítica veterinaria, a objeto de realizar vigilancia epidemiológica de ciertas enfermedades de interés en salud pública.

Este es un trabajo que aporta información epidemiológica relevante para el estado Bolívar, sobretodo de prevalencia de la infecciones por hongos endémicos. Se han realizado estudios de esta índole en áreas rurales y suburbanas del estado, pero es la primera vez que se realiza en un área urbana, como lo es el municipio Caroní.

Siendo el estado Bolívar el más grande de Venezuela, realizar este tipo de estudio en la ciudad más poblada del estado ha resultado de gran interés. Así pues, se dieron a conocer los resultados del estudio a los líderes de la comunidad y discutidos posteriormente. También se entregó a Fundacite Bolívar un informe con los resultados donde se demuestra una alta prevalencia de estas infecciones.

Los hallazgos de esta investigación indican la necesidad de promover la búsqueda activa de hongos, en especial de patógenos primarios, fundamentalmente en individuos con infecciones respiratorias crónicas, que no mejoran con terapia antibacteriana y en pacientes sintomáticos respiratorios inmunocomprometidos en zonas urbanas del estado Bolívar, así como en otras que reúnan las características ambientales y geográficas, propicias para el desarrollo de hongos patógenos sistémicos.

\section{AGRADECIMIENTOS}

A la Lic. Lucile Tessari de Calzadilla, Br. Daniela Vhalis Tovar, Licda. Irais Dimas, Licda. Ytalia Blanco, Licda. Rosario Tutaya, Licda. Johana Pinto, Consejo Comunal Bicentenario en especial a la persona Sr. Elio Medrano, MSc. Hernán Castellanos, Dr. Alexander Mansutti, Personal de Hyundai Ulsan Motors CA Pto. Ordaz (Centro de operaciones del estudio) Pto. Ordaz y a la Unidad Territorial del Ministerio para Ciencia, Tecnología e Innovación de Ciudad Guayana, por su colaboración y apoyo logístico en la realización de esta investigación.

\section{FINANCIAMIENTO}

Este trabajo fue financiado parcialmente por el Consejo de Investigación de la UDO Núcleo Bolívar, Grupo de Micología Experimental y Clínico Bolívar, Unidad de Diaǵnóstico Biomédico Bolívar CA, (UNDIBIBO, CA), Centro Clínica Familia, Electrónica Guayana, Bufete Borregales-Calzadilla y Banco del Sur de Puerto Ordaz.

\section{REFERENCIAS}

1. Ashraf N, Kubat RC, Poplin V, Adenis AA, Denning DW, Wright L, McCotter O, Schwartz IS, Jackson BR, Chiller T, Bahr NC. Re-drawing the maps for endemic mycoses. Mycopathologia 2020; 185(5):843-865.

2. Martínez D, Hernández R, Mendoza M, Alvarado $P$. Aportes recientes para el estudio de las micosis en Venezuela. Salud (i) Ciencia 2016; 22:283-286.

3. Cermeño JR, Mareano A, Sandoval M. Infecciones fúngicas en pacientes infectados por VIH en el Complejo Hospitalario Universitario "Ruiz y Páez". Bol Venez Infectol 2016; 27(2):93-99.

4. Cermeño JR, Sandoval de Mora M. Infecciones por Histoplasma capsulatum y el complejo Paracoccidioides spp. en la sede 5 de julio de la Universidad Nacional Experimental de Guayana, Ciudad Bolívar. Es- 
tado Bolívar, Venezuela. Copérnico 2014; $10(20): 24-35$.

5. Martinez R. Epidemiology of paracoccidioidomycoses. Rev Inst Med Trop Sao Paulo 2015; 57 (Suppl 19):11-20.

6. Wheat LJ, Azar MM, Bahr NC, Spec A, Relich RF, Hage C. Histoplasmosis. Infect Dis Clin North Am 2016; 30(1):207-27.

7. Martinez R. New trends in paracoccidioidomycosis epidemiology. J Fungi (Basel) 2017; 3(1):1.

8. Deepe GS Jr. Outbreaks of histoplasmosis: The spores set sail. PLoS Pathog 2018; 14(9):e1007213.

9. Shikanai-Yasuda MA, Mendes RP, Colombo AL, Queiroz-Telles F, Kono ASG, Paniago AMM, Nathan A, Valle ACFD, Bagagli E, Benard G, Ferreira MS, Teixeira MM, SilvaVergara ML, Pereira RM, Cavaleante RS, Hahn R, Durlacher RR, Khoury Z, Camargo ZP, Moretti ML, Martinez R. Brazilian guidelines for the clinical management of paracoccidioidomycosis. Rev Soc Bras Med Trop 2017; 50(5):715-740.

10. Giusiano G, Aguirre C, Vratnica C, Rojas F, Corallo T, Cattana ME, Fernández M, Mussin J, de Los Angeles Sosa M. Emergence of acute/subacute infant-juvenile paracoccidioidomycosis in Northeast Argentina: Effect of climatic and anthropogenic changes? Med Mycol 2019; 57(1):30-37.

11. Johnston L, Mackay B, King T, Krockenberger MB, Malik R, Tebb A. Abdominal cryptococeosis in dogs and eats: 38 cases (2000-2018). J Small Anim Pract 2021; 62(1):19-27.

12. Park BJ, Wannemuehler KA, Marston BJ, Govender N, Pappas PG, Chi Rajasingham R, Smith RM, Park BJ, Jarvis JN, Govender NP, Chiller TM, Denning DW, Loyse A, Boulware DR. Global burden of disease of HIV-associated cryptococeal meningitis: an updated analysis. Lancet Infect Dis 2017; $17(8): 873-881$.

13. Spec A, Powderly WG. Cryptococeal meningitis in AIDS. Handb Clin Neurol 2018; 152:139-150.

14. Zhang C, Tan Z, Tian F. Impaired consciousness and decreased ǵlucose concentration of CSF as prognostic factors in immunocompetent patients with cryptococeal meningitis. BMC Infect Dis 2020; 20(1):69. Springer DJ, Chaturvedi V.

15. Rodrigues AM, Beale MA, Hagen F, Fisher MC, Terra PPD, de Hoog S, Brilhante RSN, de Aguiar Cordeiro R, de Souza Collares Maia Castelo-Branco D, Rocha MFG, Sidrim JJC, de Camargo ZP. The global epidemiology of emerging Histoplasma species in recent years. Stud Mycol 2020; 97:100095.

16. Taborda CP, Buccheri R, Benard G, Duarte-Neto AN, Nosanchuk JD, Travassos LR. Paracoccidioides spp. and Histoplasma capsulatum: current and new perspectives for diagnosis and treatment. Curr Top Med Chem 2018; 18(15):1333-1348.

17. Mittal J, Ponce MG, Gendlina I, Nosanchuk JD. Histoplasma capsulatum: mechanisms for pathogenesis. Curr Top Microbiol Immunol 2019; 422:157-191.

18. León J. Golondrinas Migratorias Australes en Ciudad Guayana. [En línea] Agosto 2012. [Fecha de consulta: 03 enero del 2014]. Disponible en URL: https://golondrinasmigratorias.blogspot.com/p/preguntas-frecuentes.html.

19. Lentino M, Salcedo M, Malavé V. Aves del Escudo Guayanés de Venezuela. En: Lasso CA, Señaris JC (Eds.), Volumen VI. Fauna Silvestre del Escudo Guayanés (ColombiaVenezuela). Instituto de Investigáción de Recursos Biológicos Alexander von Humboldt. Serie Editorial Fauna Silvestre Neotropical: Bogotá, DC. Colombia; 2018. 283-343. [Fecha de consulta: 10 febrero del 2019]. Disponible en URL: https://www.researchgate.net/ publication/331791415_Capitulo_7_AVES_DEL_ESCUDO_GUAYANES _DE_VENEZUELA_283_Fauna_SilveStre_ del_eScudo_GuayaneS_colombia-

20. Kenzaka T, Kataoka $\bar{K}$, Fujimitsu T, Tani K. Intestinal microbiota in migrating barn swallows around Osaka. Yakugaku Zasshi 2018; 138(1):117-122.

21. Hedayati MT, Mayahi S, Fakhar M, Shokohi T, Majidi M. Cryptococcus neoformans isolation from swallow (Hirundo rustica) excreta in Iran. Rev Inst Med Trop Sao Paulo 2011; 53(3):125-127.

22. Cafarchia C, Romito D, Coccioli C, Camarda A, Otranto D. Phospholipase activity of yeasts from wild birds and possible 
implications for human disease. Med Mycol 2008; 46(5):429-434.

23. Joob B, Wiwanitkit V. Pathogenic fungi in bird excreta: A forgotten public health problem. Rev Inst Med Trop Sao Paulo 2015; 57(5):412.

24. Foti M, Rinaldo D, Guercio A, Giacopello C, Aleo A, De Leo F, Fisichella V, Mammina C. Pathogenic microorganisms carried by migratory birds passing through the territory of the island of Ustica, Sicily (Italy). Pathol aviar 2011; 40(4):405-409.

25. Ryu H, Grond K, Verheijen B, Elk M, Buehler DM, Santo Domingo JW. Intestinal microbiota and species diversity of Campylobacter and Helicobacter spp. in migrating shore birds in Delaware Bay. Appl Environ Microbiol 2014; 80(6):1838-1847.

26. Fava Netto C, Raphael A. A recao intradérmica con polisacaride do Paracoccidioides brasiliensis, na blastomicose sul Americana. Rev Inst Med Trop São Paulo 1961; 31: 161-165.

27. Cermeño JR, Sandoval M. Estudio clínicoepidemiológico de histoplasmosis y paracoccidioidomicosis en la Casa de las Doce Ventanas, sede de la Universidad Nacional Experimental de Guayana, Ciudad Bolívar. Estado Bolívar. Copérnico 2012; 17: 5-14.

28. Magalhães EM, Ribeiro Cde F, Dâmaso CS, Coelho LF, Silva RR, Ferreira EB, Rodrigues MR, Camargo ZP, Velloso TR, Malaquias LC. Prevalence of paracoccidioidomycosis infection by intradermal reaction in rural areas in Alfenas, Minas Gerais, Brazil. Rev Inst Med Trop São Paulo 2014; 56(4):281-285.

29. Fornajeiro N, Ferrarezi M, Takahachi G, Estivalet T. Inquérito epidemiológico sobre a paracoccidioidomicose utilizando a ǵp43 em dois municípios do noroeste do Paraná, Brasil. Rev Soc Bras Med Trop 2005; 38(2):191-193.

30. Marques AP, Oliveira SM, Rezende GR, Melo DA, Fernandes-Fitts SM, Pontes ER. Evaluation of Paracoccidioides brasiliensis infection by the gp43 intradermal test in rural settlements in Central-West Brazil. Mycopathology 2013; 176:41-47.

31. Kwong-Chung K, Bennett JE. Medical Mycology. Philadelphia, Lea and Febiger, 1992. 866 pp.
32. Hoog G, Guarro J, Gene J, Figueras M. Atlas of clinical fugí. $2^{\text {da }}$ edition. Barcelona, España: Universitat Rovira; 2000. 1126 pp.

33. Kwon-Chung KJ, Bennett JE. Epidemiologic differences between the two varieties of Cryptococcus neoformans. Am J Epidemiol 1984; $120(1): 123-130$.

34. Casadevall A, Perfect JR. Cryptococcus neoformans. Washington D.C. ASM Press, 1998. 542 pp.

35. Kwon-Chung K, Polacheck I, Bennett J. Improved diagnostic medium for separation of Cryptococcus neoformans var. gattii (Serotypes A and D) and Cryptococcus neoformans var. gattii (Serotypes B and C). J Clin Microbiol 1981; 15:535-537.

36. Conly, G. Manual of standardized serodiagnostic procedures for systemic mycoses: part I: agar immunodifussion test. In PAHO. Scientific Publication 1972; 307:1.

37. Meridian Bioscience, Inc. Fungal Inmunodiffusion system. For in vitro diagnostic use only. Catalog $\mathrm{N}^{\circ} 100100.2003 ; 11: 22-28$.

38. Cermeño JR, Cermeño JJ, Gerardo G. Hernández Y, Orellán Y, Blanco Y, Salvador Penna S, García L, Mender T, Gonsálvez M, López C, Hernández N, Longa I, Gottberg E, Basanta A, Castro M, Millán I, León W, Plaz F, Jahouhari C, Cabello I. Epidemiological study of paracoccidioidomycosis and histoplasmosis in a suburb of San Félix city, Bolívar state, Venezuela. Invest Clín 2009; 50:213-220.

39. Cermeño JR, Hernández I, Cermeño JJE, Godoy G, Cermeño JJO, Orellán Y, Blanco Y, Cabello I, Guzmán Y, Alcalá F, García T, Penna S. Epidemiological survey of histoplasmine and paracoceidioidine skin reactivity in an agricultural area in Bolívar state, Venezuela. Eur J Epidemiol 2004; 19:189193.

40. Cermeño JR, Cermeño JJ, Hernández I, Godoy G, Cermeño JJ, Cabello I, Orellán Y, Blanco Y, Penna S. Histoplasmine and paracoccidiodine epidemiological study in Upata, Bolívar state, Venezuela. Trop Med Int Health 2005; 10(3):216-219.

41. Silva-Vergara M, Martínez R. Inquérito epidemiológico com paracoceidoidina e histoplasmina em área aǵrícola de café em Ibiá, Minas Gerais, Brasil. Rev Iberoam Micol 1998; 15(4):294-297. 
42. Albornoz MB, Albornoz R. Estudio de la sensibilidad específica en residentes de un área endémica a la paracoccidioidomicosis en Venezuela. Mycopathol Mycol Appl 1971; 45:65-75.

43. Mirt JA, Mirt SA. Estudio de la paracoccidioidomicosis en el estado Falcón. Venezuela. Casos clínicos y encuesta epidemiológica con paracoccidioidina en un área endémica. Boletín Informativo "Las Micosis en Venezuela" 1986; 4:16-17.

44. Kalmar EM, Alencar FE, Alves FP, Pang LW, Del Negro GM, Camargoo ZP. Paracoccidioidomycosis: an epidemiologic survey in a pediatric population from the Brazilian Amazon using skin tests. Am J Trop Med Hyǵ 2004; 71:82-86.

45. A van Gelderen de Komaid, E Durán, I Borges de Kestelman. Histoplasmosis and paracoccidioidomycosis in northwestern Arǵentina III. Epidemiological survey in Vipos, La Toma, and Choromoro - Trancas, Tucumán, Arǵentina. Eur J Epidemiol 1999; 15(4):383-388.

46. Fava S, Fava Netto C. Epidemiologic surveys of histoplasmin and paracoccidioidin sensitivity in Brazil. Rev Inst Med Trop São Paulo 1998; 40:155-164.

47. Anacona C, González C FE, Vásquez-A LR, Escandón P. First isolation and molecular characterization of Cryptococcus neoformans var. grubii in excreta of birds in the urban perimeter of the Municipality of Po- payan, Colombia. Rev Iberoam Micol 2018; 35(3):123-129.

48. Ghaderi Z, Eidi S, Razmyar J. Highh Prevalence of Cryptococcus neoformans and isolation of other opportunistic fungi from pigeon (Columba livia) droppings in Northeast Iran. J Avian Med Surǵ 2019; 33(4):335-339.

49. Pakshir K, Fakhim H, Vaezi A, Meis JF, Mahmoodi M, Zomorodian K, Javidnia J, Ansari S, Hagen F, Badali H. Molecular epidemiology of environmental Cryptococcus species isolates based on amplified fragment length polymorphism. J Mycol Med 2018; 28(4):599-605.

50. Rosario I, Acosta B, Colom F. La paloma y otras aves como reservorio de Cryptococcus spp. Rev Iberoam Micol 2008; 25 (1): S13-S18.

51. Norkaew T, Ohno H, Sriburee P, Tanabe K, Tharavichitkul P, Takarn P, Puengchan T, Bumrungsri S, Miyazaki Y. Detection of environmental sources of Histoplasma capsulatum in Chiang Mai, Thailand, by nested PCR. Mycopathologia 2013; 176(5-6):395402.

52. Cermeño JR, Hernández I, Cabello I, Orellán Y, Cermeño JJ, Albornoz R, Padrón E, Godoy G. Cryptococcus neoformans and Histoplasma capsulatum in dove's (Columbia livia) excreta in Bolívar state, Venezuela. Rev Latinoam Microbiol 2006; 48(1):6-9. 